J. Neurol. Neurosurg. Psychiat., 1955, 18, 97.

\title{
THE ARTERIAL SUPPLY OF THE SPINAL CORD AND ITS SIGNIFICANCE
}

\author{
BY \\ D. H. M. WOOLLAM and J. W. MILLEN \\ From the Department of Anatomy, Cambridge
}

It has long been recognized that an adequate blood supply is an essential prerequisite to the normal functioning of the neuron. The major contributions to the study of the vascular architecture of the central nervous system have naturally been based on the examination of the brain. Investigations of the arterial supply of the spinal cord have been few, and have been limited to the examination of human material obtained at necropsy (Adamkiewicz, 1882 ; Kadyi, 1889 ; Tureen, 1938 , Suh and Alexander, 1939). It appeared therefore that a study of the arterial patterns of the spinal cord based primarily on the use of an experimental animal might serve to elucidate the principles on which the blood vessels are distributed to the spinal cord, at the same time providing additional information concerning the manner of vascularization of the central nervous system in general.

A major obstacle to the study of the vascular arrangements of the central nervous system has been the problem of differentiating the arteries from the veins. Indeed, as recently as 1938, Campbell was obliged to point out that the extensive monograph of Pfeifer (1930) on the angio-architecture of the cerebral cortex suffered from the serious defect that the structures described as veins were in reality arteries and vice versa. The first experimental method which allowed of the certain differentiation of the cerebral arteries and veins was that introduced by Scharrer (1940). In this method the arterial system was injected with a gelatin solution incorporating rice starch, the particle size of which prevented the injection mass from entering the capillaries. Thick sections of the brain treated in this way were immersed in iodine solution, the starch-iodine reaction took place, and the arteries appeared deep black. Using the same technique, the venous system of a similar animal was filled and the results compared.

Scharrer's method, although allowing a clear distinction to be made between arteries and veins, does not enable the whole vascular system, including the capillaries, to be visualized in the same organ. In a previous paper (Millen and Woollam, 1953) a method was described which allows such a differentiation to be made. Briefly the technique was as follows. Blue and red dispersion media were used for the injection. (These media were supplied by the B.B. Chemical Co., Ltd., Ulverscroft Road, Leicester, England, who state that the dispersions were prepared by ball-milling pigments with a small quantity of a highly active surface wetting agent. The blue pigment used was copper phthalocyanine, and the red was a red lake pigment.) When the red pigment was introduced into the heart or ascending aorta it filled the arteries and arterioles, passed on into the capillaries, and filled both the capillaries and veins. The introduction of the blue pigment immediately afterwards at the same site pushed the red pigment on into the capillaries and veins, but the blue pigment did not itself enter the capillaries. By this method a picture of the whole vascular system of the animal could be obtained, in which the arteries were outlined by blue pigment and the veins and capillaries by red. This method was used for the study of the arterial patterns in the rat's spinal cord which is the subject of the present communication.

\section{Materials and Methods}

The spinal cords of 16 adult rats were examined. In each case the rat was killed with ether, and $2 \mathrm{ml}$. of the red dispersion was injected into the ascending aorta through the heart at a pressure of about $80 \mathrm{~mm}$. $\mathrm{Hg}$. As soon as this injection was completed, $3 \mathrm{ml}$. of the blue dispersion was introduced in a similar fashion. After fixation in $10 \%$ formalin for a week, the spinal cord of the animal was dissected free from the surrounding structures and the surface distribution of the blood vessels studied and photographed in colour. Sections were then cut with the freezing microtome at 100 to $200 \mu$ in the transverse, sagittal, and coronal planes at each level of the spinal cord. These sections were rapidly dehydrated in ascending grades of alcohol, cleared in xylol, and mounted in balsam.

In addition, a number of dissecting-room specimens 
of human spinal cords were examined. In all of these the arteries were outlined by the injection of red lead.

\section{Findings}

The method employed in this investigation enabled a clear distinction to be made between the arteries and veins, for the arteries were filled with the blue dispersion and the veins with the red. The difference in the morphology of the vessels was exactly similar to that which Scharrer (1944) described in the intracerebral vessels, for the arteries ran in smooth, graceful curves and gave off their branches at acute angles, whereas the veins received their tributaries at more obtuse angles.

The spinal cord of the rat receives its major arterial supply from a number of radicular arteries which run along the ventral nerve roots to reach the anterior surface of the cord. Each of these radicular vessels divides near the midline into an ascending and a descending branch (Fig. 1). The ascending and descending branches form a midline chain of anastomoses which run the whole length of the cord from the medulla to the beginning of the filum terminale. The uppermost ascending branch is joined by a descending vessel formed by the union of branches from the vertebral arteries (Fig. 2).

The radicular vessels are derived from arteries in the neighbourhood of the vertebral column and are comparatively constant in their arrangement. Three or four large radicular arteries supply the cervical region of the spinal cord, but, in the thoracic region, the radicular arteries are small in size, inconstant in arrangement, and only two or three in number. In the lumbar region, an extremely large vessel invariably runs along the second or third lumbar nerve root. This vessel is unilateral and most often enters from the right side. It appears to be the sole arterial supply to the whole of the lower lumbar and sacral parts of the spinal cord. This large lumbar radicular artery shows clearly the usual divisions into ascending and descending branches. In the case of this vessel the division occurs quite some distance from the midline and the descending branch is much larger than the ascending branch (Fig. 3).

In the cervical region the anastomoses between the adjacent branches of the radicular vessels are well marked, and the anastomotic chain has at first glance the appearance of a median artery continuing downwards from the anterior spinal vessel formed by the branches from the vertebral arteries. On more careful examination, however, its composition from the ascending and descending branches of the radicular arteries is apparent.

The ascending and descending branches of the thoracic radicular arteries are slender vessels which run considerable distances before anastomosing with each other or with the descending branch of the lowest cervical artery or the small ascending branch of the great lumbar radicular artery (Fig. 1). In consequence the anastomoses between these vessels are of an exiguous nature, and the anterior anastomotic chain is poorly defined and in some instances almost discontinuous over the thoracic part of the spinal cord.

The arrangement of the arterial supply to the anterior surface of the spinal cord of the rat (Fig. 4) may be summarized as follows :-

The anterior surface of the cord is supplied by radicular arteries reaching the cord along the ventral nerve roots. Each of these arteries divides into an ascending and a descending branch, and is primarily responsible for the blood supply to a particular region of the cord. The ascending and descending branches anastomose freely in the cervical region but only to a very slight extent in the thoracic region. The lumbar and sacral parts of the cord are supplied by the branches of the single great lumbar radicular vessel. The smaller ascending branch of this vessel anastomoses poorly with the lowest thoracic descending branch.

Only a small number of human spinal cords have so far been examined. These showed that the arrangement of the radicular vessels is essentiallyo similar to that described for the rat. The vesser described as the anterior spinal artery is in fact a longitudinal anastomotic chain formed by the ascending and descending branches of the radicular arteries in a manner similar to that observed in the rat, rather than a continuous vessel coursing down the anterior midline from the medulla to the filum terminale. The anastomoses in the thoracic region are also, as in the rat, extremely slender.

In each segment of the rat's cord, central branches pass, from the ascending and descending branches of the radicular arteries, into the anterior median fissure to enter the substance of the spinal cord. Because of the distance of the bifurcation of the great lumbar vessel from the midline, some of the central branches of this vessel can be seen coursing over the anterior surface of the cord to gain the anterior fissure (Fig. 3). In general, however, the central branches pass directly downwards into the anterior fissure and have no course outside the nervous tissue. The central branches, as seen in the series of coronal sections, enter the bases of the anterior horns of the grey matter and usually pass alternately to the right and left sides (Fig. 5). On entering the grey matter each vessel breaks up into arterioles which are distributed to the anterior 


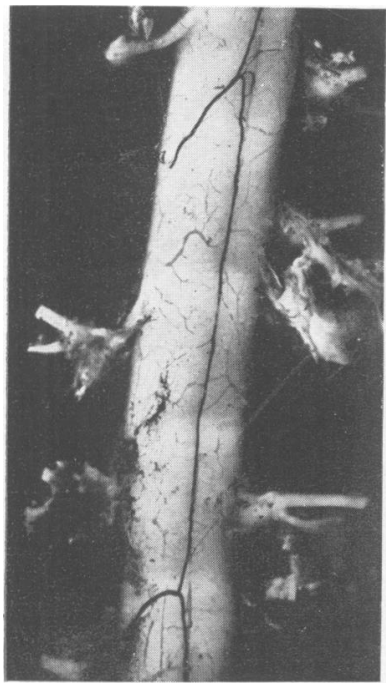

FIG. 1.

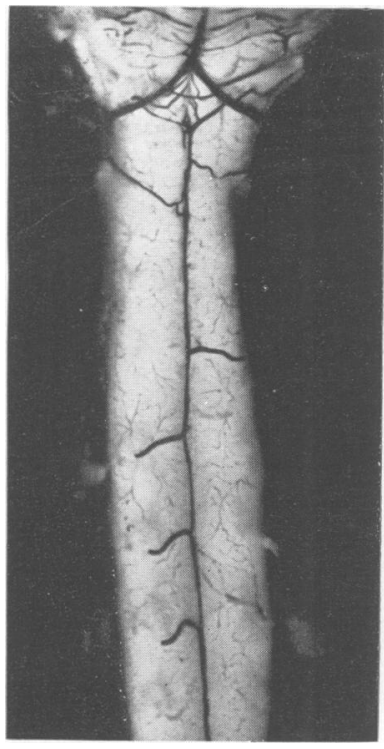

FIG. 2.

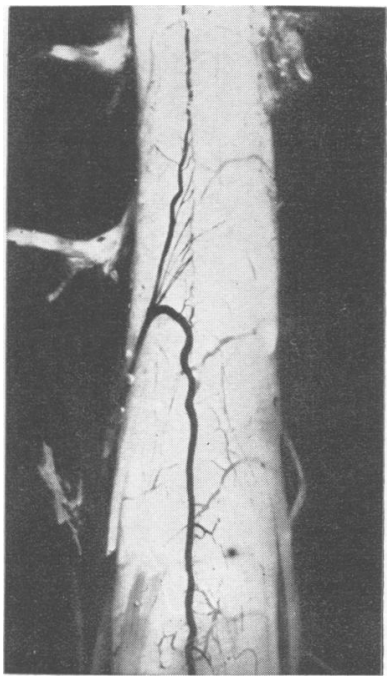

FIG. 3.

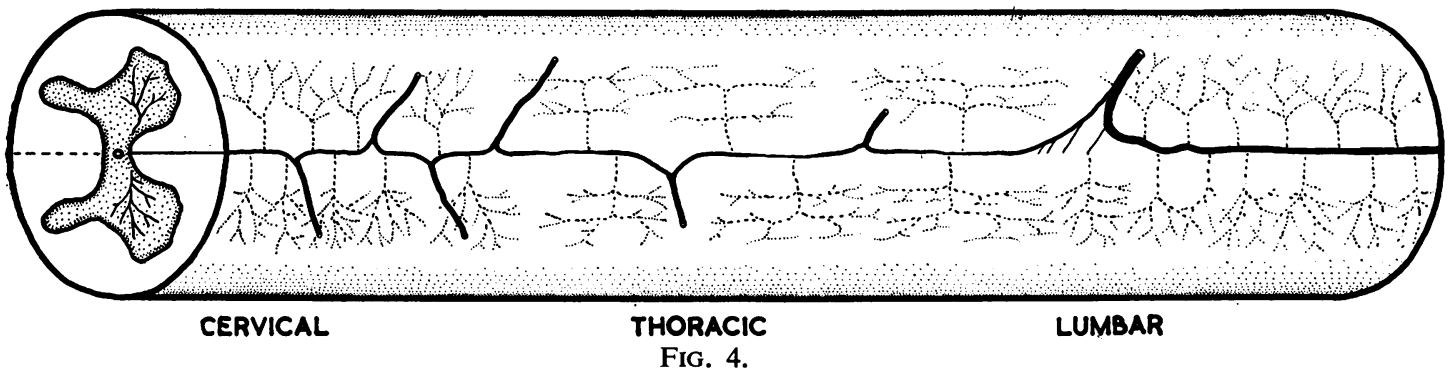

FIG. 4.

Fig. 1.-Anterior surface of injected spinal cord of a rat. Thoracic region. Two radicular arteries are shown dividing into ascending and descending branches. $\times 4$.

Fig. 2.-Anterior surface of medulla and cervical region of injected spinal cord of a rat, showing the contributions from the vertebral arteries to the midline anastomotic chain. $\times 4$

Fig. 3.-Anterior surface of injected spinal cord of a rat. Lumbar region. The large radicular artery, corresponding to the artery of Adamkiewicz in man, is shown dividing into its two branches. Central branches run on the surface of the cord towards the anterior median fissure. $\times 4$.

Fig. 4.-Diagram illustrating the arrangement of the anterior radicular arteries, the midline anastomotic chain, and the distribution of the central arteries in the spinal cord of the rat.

Fig. 5.-Coronal section through the cervical region of the injected spinal cord of a rat, showing the alternate distribution of the central branches. $\times 16$.

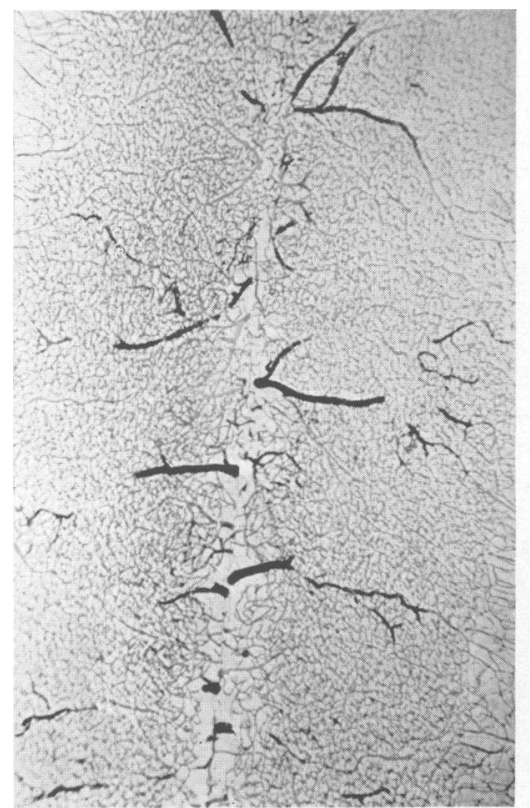

FIG. 5. 


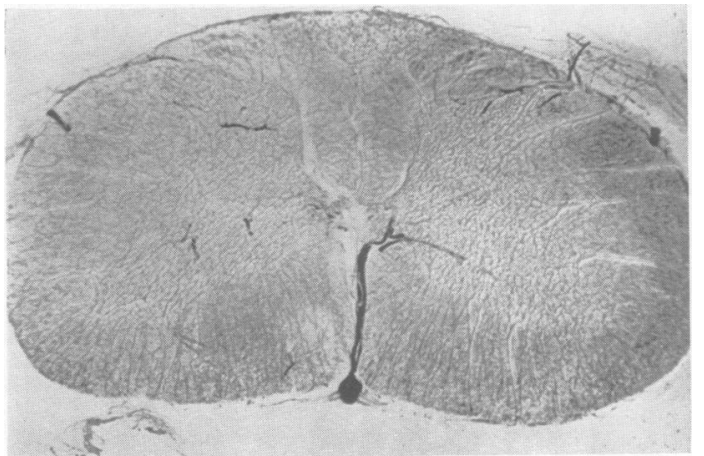

Fig. 6..

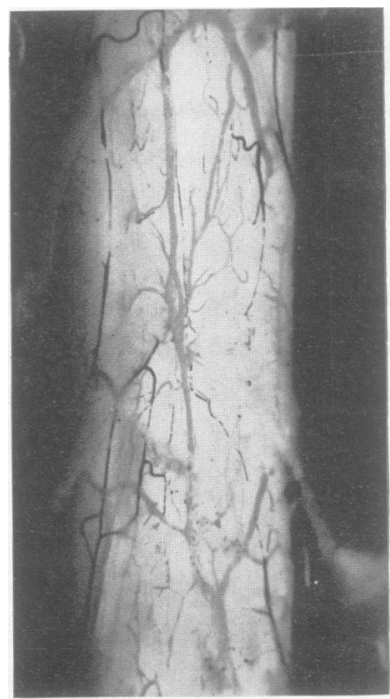

Fig. 7.

and lateral horns and the basal part of the posterior horn (Fig. 6).

The density of distribution of these central branches varies from region to region of the cord. Thus in $1 \mathrm{~cm}$. of the cervical cord 12 central branches are distributed to each side ; in $1 \mathrm{~cm}$. of the lumbar cord 17 branches go to each side, whereas in the same length of the thoracic cord only seven central branches are given off on each side. Because of the long distance separating the central branches in the thoracic region, their manner of distribution differs from that of the other regions. In the lumbar and cervical regions the branches are distributed in a compact, bushy manner, whereas in the thoracic region they trail away horizontally and are less closely packed (Fig. 4).

In addition to the anterior anastomotic chain, there are two smaller anastomotic chains situated on the posterior aspect of the rat's spinal cord in

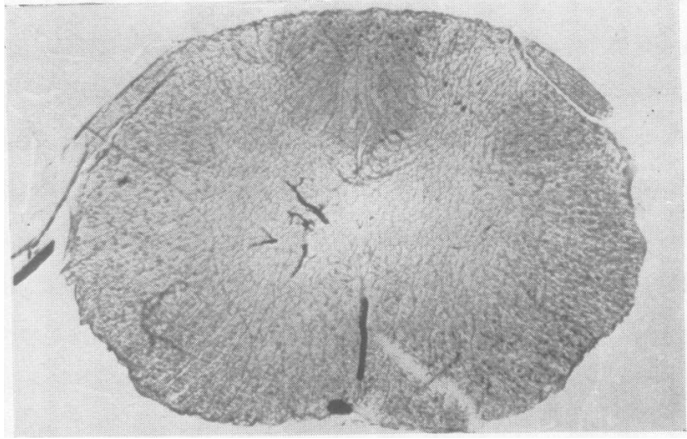

Fig. 6b.

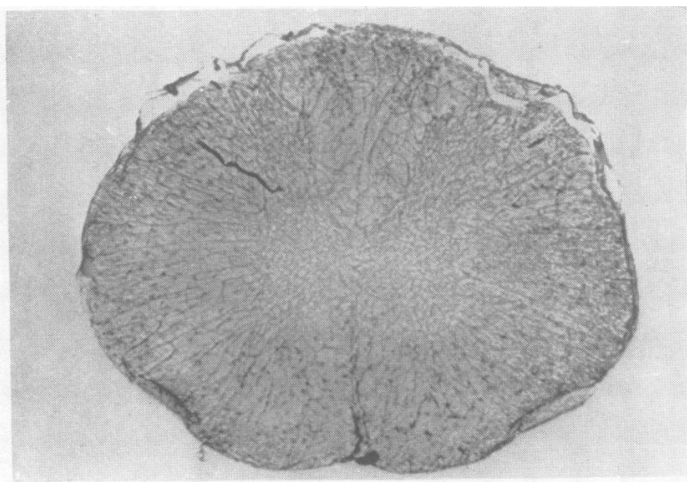

Fig. 8.

Fig. 6. -Transverse sections through the injected spinal cord of rat. $\times 16$. (a) central artery running in the anterior median fissure to the base of the anterior horn; $(b)$ the distribution of the central branch in the substance of the grey matter.

Fig. 7.-Posterior surface of the injected spinal cord of a rat. The anastomoses between the posterior radicular arteries are in the form of irregular loops. $\times 7$.

Fig. 8.-Transverse section through the injected spinal cord of a rat, showing a branch from a posterior radicular arte:y entering the posterior horn of the grey matter. $\times 16$

relation to the dorsal nerve roots. These are much less constant in their situation than the anterior anastomotic chain, and they form irregular loops between each other and in their individual courses (Fig. 7). They are formed by radicular vessels which arise from the arteries in the neighbourhood of the vertebral column and reach the postero-lateral sulcus by passing along the dorsal nerve roots. Central branches arise from these posterior arteries and pierce the tip of the posterior horn of grey matter (Fig. 8) to supply that part of the posterior horn which is not supplied by the central branches of the anterior vessels. The central branches of the posterior arteries are very restricted in their distribution and travel upwards and downwards only to $\mathcal{N}$ a limited extent. The density of distribution of $N$ these branches in the various parts of the cord $N$ closely parallels that of the anterior central branches. 


\section{Discussion}

There are no descriptions in the literature of the arterial supply of the spinal cord in mammals other than man, but in current textbooks of human anatomy an anterior spinal artery is described which starts above by the union of branches from the vertebral arteries and traverses the cord to its lower extremity, being reinforced by a succession of small contributions from vessels in the neighbourhood of the spinal cord. In such descriptions the main emphasis is placed on the median artery on the anterior surface of the spinal cord, and the radicular arteries are relegated to a secondary position. In the rat, as the illustrations show, there can be little doubt that there is an arterial anastomotic chain formed entirely by the anterior radicular arteries with the exception of the upper cervical part of the chain which is formed by the anterior spinal branches of the vertebral arteries. Each of these anterior radicular arteries divides into ascending and descending branches which anastomose with a branch from the next significant radicular artery above and below. The examination of the human spinal cord made during the course of this investigation suggested that a similar arrangement is present in man, and in this respect amplified the views of Suh and Alexander (1939). The clearest illustration of the manner in which the anterior anastomotic chain is formed is seen in the illustration of the large lumbar radicular artery of the rat (Fig. 3), which corresponds to the arteria radicalis anterior magna described by Adamkiewicz (1882) in man. The fact that central branches supplying the grey matter of the spinal cord are given off from this artery from the point of its division well away from the midline leaves little doubt that it represents the equivalent in the rat of the artery described as the "anterior spinal artery" in most textbooks of human anatomy. The examination of human material made during the course of this investigation confirmed that the arrangements are essentially similar, and indicated that the lower thoracic, lumbar, and sacral regions of the cord receive their blood supply from the ascending and descending limbs of the arteria radicalis anterior magna of Adamkiewicz which is the "anterior spinal artery " of anatomical textbooks or " truncus arteriosus anterior" of Suh and Alexander (1939) in this situation. Furthermore this particular artery serves to show to a marked extent a point, which is also borne out by examination of the thoracic and cervical regions of the cord, that the arterial supply of the spinal cord is from a series of individual arteries which anastomose with each other rather than from a "truncus arteriosus anterior" re- plenished by a succession of small radicular contributions.

The question arises as to the normal direction of blood flow in the midline anastomotic chain. Inferiorly there can be little doubt that the blood must flow upwards and downwards respectively in the ascending and descending branches of the artery of Adamkiewicz, so that from the point of division of this artery blood flows downwards to the lumbo-sacral enlargement of the cord and upwards to the thoracic region. Superiorly the vertebral artery corresponds very closely to the artery of Adamkiewicz, differing largely in the fact that it is bilateral. Its large ascending branch meets that of the opposite side to form the basilar artery and its descending division joins its fellow of the other side to form the anterior spinal artery, in which the blood must flow downwards to the cervical region of the cord to meet blood entering the anastomotic chain in the ascending branch of the first significant cervical radicular artery. It is evident therefore that the blood flow in the midline anastomotic chain is by no means all in one direction, from above downwards, but that it flows in opposite directions at different levels. It follows from this that several water-sheds are produced at points more or less equidistant from the point of division of the large radicular arteries into ascending and descending branches. Such water-sheds are most evident in the mid-thoracic portion and at the junction of the thoracic cord with the cervical and lumbar regions.

Experimental injections into the vessels of the human spinal cord have produced curious and inconsistent findings. Thus, when making injections into the human spinal cord at various levels, Tanon (1908) found that, whereas he could fill the vascular system of the entire cord from injections made into the lumbar vessels, injections into the thoracic portion above the ninth segment filled only small stretches, and in the cervical region the territory injected from each artery was only slightly larger. On the other hand Suh and Alexander (1939) found that injections from above could only be made into the cervical region and from below only into the lumbar and thoracic regions. They considered that this was because the anastomoses between the arteries of the cervical and upper thoracic regions were so thin as to be inadequate. Apart from possible criticisms of the technique and injection media employed, the discrepancies between these observations may well be explained by the effects of post-mortem clotting in the vessels and the trauma inevitably associated with the removal of the spinal cord from the cadaver. It is not unreasonable 
therefore to conclude that it is undesirable for inferences to be drawn as to the normal direction of blood flow in the midline anastomotic chain from the behaviour of injection media introduced after death at a single point into the vascular system of the isolated human spinal cord.

In the thoracic part of the spinal cord of the rat, the midline arterial anastomosis is not only narrow with few slender radicular contributions, it also sends relatively few central branches into the substance of the cord. This arrangement is similar to that described in the human spinal cord by Suh and Alexander (1939) and Herren and Alexander (1939). Because of this arterial pattern Suh and Alexander (1939) have suggested that the vulnerability of the thoracic region of the spinal cord in certain diseases is due to the fact that the thoracic region has a relatively poor blood supply. Scharrer (1944) sought to explain the early vulnerability of the hippocampus in carbon monoxide poisoning by the rake-like arrangement in which the arteries supplying the hippocampus come off the main stem. $\mathrm{He}$ believed that in the rake-like system there tended to be a fall in pressure towards the end of the main artery, whereas in the dichotomous system the pressure was even throughout the system of ultimate branches. The diagram (Fig. 4) shows how the situation of the thoracic cord resembles that of the hippocampus, and it may well be argued that the blood pressure is lower in the thoracic part of the cord than in the other parts. Suh and Alexander (1939) believed that the early susceptibility of the thoracic cord in subacute combined degeneration could be explained by the anoxic effect of pernicious anaemia on the nervous system, and the consequent early involvement of those parts where the blood pressure was lowest. The lesions, however, which appear in the thoracic cord in this disease are lesions of the fibre tracts, and the capillary bed appears to receive no arterioles in the white matter, the white matter being supplied from the capillary bed of the grey matter. If anaemia was the cause of the damage to the white matter, one would expect the grey matter adjoining it to be equally affected. It would therefore seem unlikely that the pathology of subacute combined degeneration of the cord can readily be explained on a vascular basis.

Gerard and Serota (1936) have concluded that the amount of blood reaching a neuron varies with the degree of activity of the part of the body it supplies. The arterial supply of the spinal cord of the rat seems admirably designed to fit this principle. The cervical and lumbar regions of the cord which subserve the regions capable of the greatest activity, the limbs, are supplied with major arteries catering for their specific requirements, while the thoracic region may perhaps be regarded as taking the blood that is left over from both. Although it is obviously justifiable to comment on the manner in which a concentration of blood in the cervical and lumbar regions of the cord is achieved, there is no reason to assume as a natural corollary that the blood supply of the thoracic region is inadequate.

The examination of the arterial patterns of the spinal cord of the rat reveals two significant features. First there is the fact that the motor neurons of the spinal cord are all supplied from the same anterior anastomotic chain. Secondly there is the manner in which the arterial supply of the spinal cord of the rat exactly parallels that of man. This second observation suggests that the spinal cord of the rat affords an excellent site for study and experiment upon the general significance of the vascular supply to the central nervous system.

\section{Summary}

The arterial patterns of the spinal cord of the rat $\overrightarrow{0}$ were studied by means of a technique in which, $\dot{T}$ from an injection into the aorta, the arteries were $\mathbb{Q}_{\overrightarrow{0}} \vec{v}$ filled with a blue dispersion and the veins and $\stackrel{\circ}{\mathrm{O}}$ capillaries with a red. The arterial supply of the $\frac{}{\sigma}$ rat's spinal cord was found to bear a close resem- 3 blance to that of the human cord. From a midlineo anterior anastomotic chain formed by anteriors radicular arteries central branches supply theo anterior and lateral horns and the basal parts of the posterior horns of grey matter. This supply is less rich in the thoracic region than it is in the cervical and lumbar regions of the cord, a finding which may be related to the differing metabolic requirements of the various regions.

We wish to express our gratitude to Professor J. D. Boyd for his advice in the preparation of this paper. Our thanks are also due to Mr. T. R. L. Brooks for the photographic work.

\section{REFERENCES}

Adamkiewicz, A. (1882). S.B. Akad. Wiss. Wien. Math-nat. Kl Abt 3, 85, 101 .

Campbell, A. C. P. (1938). Res. Publ. Ass. nerv. ment. Dis., 18, 69.

Gerard, R. W., and Serota, H. (1936). Amer. J. Physiol., 116, 59.

Herren, R. Y., and Alexander, L. (1939). Arch. Neurol. Psychiat., Chicago, 41, 678 .

Kadyi, H. (1889). Über die Blutgefässe des menschlichen Rückenmarkes. Gubrynowicz and Schmidt. Lemberg.

Millen, J. W., and Woollam, D. H. M. (1953). J. Anat., Lond., Pfeifer,
Angioarchitektonik des menschlichen Gehirns. Angioarch.

Scharrer, E. (1940). Anat. Rec., 78, 173.
Quart. Rev. Biol., 19, 308.

Suh, T. H., and Alexander, L. (1939). Arch. Neurol. Psychiat., Chicago, 41, 659 .

Tanon, L. (1908). Thèse de Paris, No. 98. Vigot Frères, Paris

Tureen, L. L. (1938). Res. Publ. Ass. nerv. ment. Dis., 18, 394. 